\title{
Antiestrogen Therapy
}

National Cancer Institute

\section{Source}

National Cancer Institute. Antiestrogen Therapy. NCI Thesaurus. Code C15757.

Drug treatment to block the action of the female hormone estrogen. 